Chapter 17

\title{
Aortic Valve Replacement for Calcified Aortic Valves
}

\author{
Kazumasa Orihashi \\ Additional information is available at the end of the chapter \\ http://dx.doi.org/10.5772/53418
}

\section{Introduction}

Valve replacement has been the standard treatment for aortic stenosis until the development of transcatheter aortic valve implantation (TAVI). Although TAVI provides a treatment with fairly acceptable outcomes for patients with high surgical risk, aortic valve replacement remains essential even in the TAVI era, because surgical treatment is indicated when TAVI cannot be performed due to a small aortic annulus or inappropriate access route. In addition, surgical treatment may be necessary when complications develop during TAVI procedures. Therefore, a more meticulous procedure is required for surgeons. With an increasing number of elderly patients who need surgical treatment and are at high risk due to aging, comorbidities, or medications such as steroids or antiplatelet drugs, trivial pitfalls during surgery can lead to catastrophic results. Furthermore, many patients with hemodialysis and marked systemic calcification require aortic valve surgery in Japan [1].

Complications encountered during surgery for aortic stenosis can be associated with catastrophic events such as myocardial infarction, cerebral embolism or aortic dissection. This is because a calcified aortic valve rarely exists alone, but is often associated with marked and diffuse calcification in the aorta, coronary arteries, mitral valve or even cerebral vessels [2]. The goal of surgical treatment is to implant a prosthetic valve of adequate size in each individual patient without perivalvular leak, while avoiding undesirable complications such as stroke, cardiac events or bleeding. This chapter is devoted to the tips and pitfalls in aortic valve replacement of calcified aortic valves with a discussion of preoperative and intraoperative strategies to achieve the best possible outcomes. 


\section{Calcified aortic valve}

\subsection{Etiologies of calcification}

There are three main etiologies of aortic stenosis. The location and extent of calcification in the aortic valve varies depending on its etiology (Fig. 1). In rheumatic valvular disease, there is initial thickening and fusion of the cusps with later involvement of the annulus. The fusion of cusps results in the formation of a valve orifice that looks like a fish mouth. There is also severe mitral valve calcification in patients with combined valvular disease. In arteriosclerotic aortic stenosis, calcification is most prominent in the annulus, sinus of Valsalva and ascending aorta. Calcification of the cusps and calcium deposits on them markedly limit cusp opening. In bicuspid aortic stenosis, disease on the annulus is rather mild but fusion of the commissures reduces the valve orifice area and forms a slit-like orifice. Calcification is often present in bicuspid aortic stenosis, but is rather mild compared with that in arteriosclerotic aortic stenosis.

A

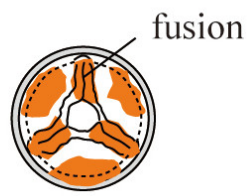

rheumatic disease
B

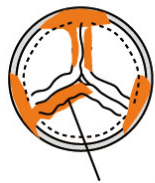

calcified cusps
C

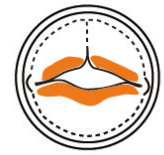

bicuspid valve

Figure 1. Etiologies of aortic stenosis. A: Rheumatic disease initialy occurs on the cusps and extends toward the annulus. Fusion of the cusps causes a stenotic orifice like a fish mouth. B: Arteriosclerotic disease extends from the aorta toward the cusps. Opening of the cusps is often disturbed by rigidity of the cusps and calcium deposits on them. C: In bicuspid valve, calcification takes place mainly on the cusps.

\subsection{Removal of calcium}

A calcified aortic valve can be a cause of arterial embolism resulting in stroke or myocardial infarction [3-5]. A mobile mass is occasionally found attached to the calcified portion of the aortic valve. A calcified aortic valve as well as the presence of a mobile mass can be assessed by transthoracic echocardiography. Computed tomography (CT) is suitable for precisely assessing the presence and extent of calcification in the annulus and the entire aorta [6,7]. As the calcified cusps are resected, calcium in the annulus needs to be adequately removed to allow subsequent sutures for valve implantation. Calcium deposits are hard but easily crumble into pieces. They can be crushed by clamps and removed or can be fragmented by a Cavitron Ultrasound Surgical Aspirator (CUSA). Either way, loosened calcium needs to be carefully eliminated to prevent it from entering the left ventricle and causing stroke, or entering the left coronary artery and causing myocardial infarction. Digital palpation for irregularity and 
rigidity is helpful for evaluating the adequacy of calcium removal. To minimize the chance of perivalvular leakage following valve implantation, the inner aspect of the annulus should be smooth with the sewing cuff of the prosthetic valve sealing the small gaps between the sutures.

However, excessive removal of calcium may result in perforation of the aortic root or damage of the mitral valve [8]. Fig. 2 summarizes intraoperative transesophageal echocardiography (TEE) assessment and several measurements on the calcified aortic valve [9].

The extent of calcification should be carefully checked in the annulus and the aorta (described later). It is important to measure the dimensions of the annulus and sinotubular junction. When the latter is equal to or smaller than the former, difficult insertion of the prosthesis may be encountered. Although the valve can be narrowly inserted by distorting the bioprosthetic valve or the aorta, calcification in the aorta or sinotubular junction makes the latter difficult.

Acoustic shadow indicates the presence and extent of calcification. Fig. 3 shows three different degrees of calcification in the annulus of the noncoronary cusp. When it is transmural or continues to the anterior mitral leaflet as shown in Fig. 3C, excessive removal of calcium should be avoided and limited to a reasonable depth.

A
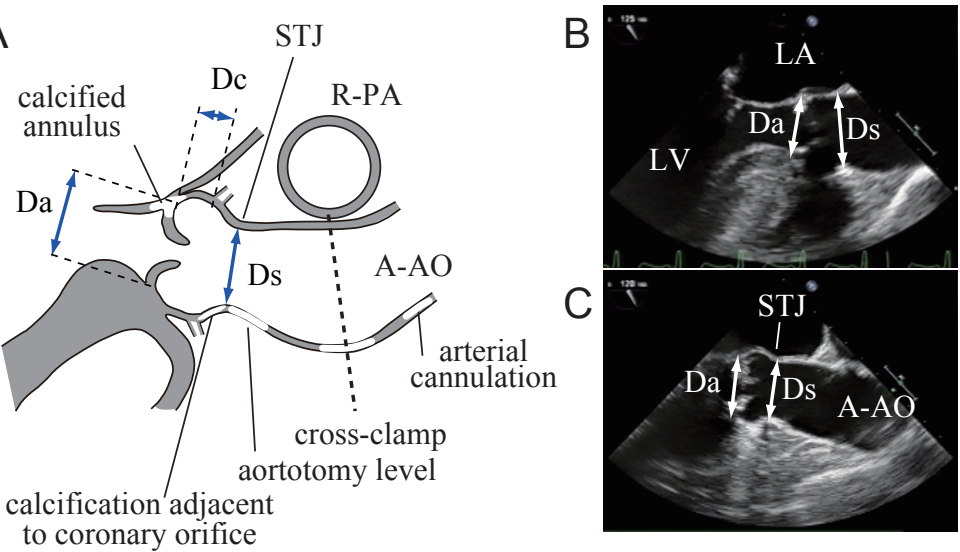

Da: annular diameter

Ds: diameter of STJ

Dc: distance

between annulus and coronary orifice

Figure 2. Intraoperative transesophageal echocardiography (TEE) assessment. A: Calcification in the annulus, sinus of Valsalva, and A-AO is assessed. Important measurements are shown. Annular diameter (Da) is usually smaller than the diameter of STJ (Ds) (B). When Ds is equal or smaller than Da (C), difficult insertion of prosthetic valve may be encountered. (modied from reference 9) 

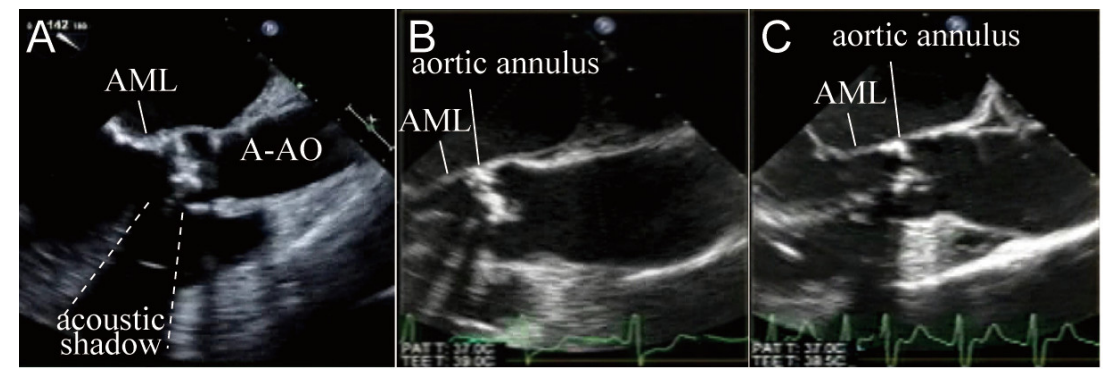

Figure 3. Calcification in the aortic annulus. A: Calcification is present on the cusps, accompanied by acoustic shadow but not in the annulus. B: Calcification in the annulus. C: Calcification extends to the AML.

\subsection{Optimizing valve size}

A calcified aortic valve limits the size of the prosthetic valve that can be implanted. When the aortic annulus is too small, patient-prosthesis mismatch can arise that may lead to sustained pressure overload of the left ventricle and subsequent chronic heart failure. A bioprosthetic valve is desirable for elderly patients with poor anticoagulation compliance to minimize the risk of cerebral infarction, or in patients that need antiplatelet therapy (patients with stroke or coronary stents); however, there are occasions where only a small-sized mechanical valve can be inserted due to a small aortic annulus [19]. Several solutions are illustrated in Fig. 4.

\subsubsection{Annular dilatation}

To enlarge the annulus, the aortotomy incision line is extended proximally toward the commissure between the left and non-coronary cusps or toward the noncoronary sinus of Valsalva (Fig. 4A) [11]. However, a markedly calcified annulus and/or sinus of Valsalva makes this procedure difficult or even increases the risk of uncontrollable bleeding at the suture line. Preoperative CT assessment or intraoperative TEE is helpful for making a decision.

A

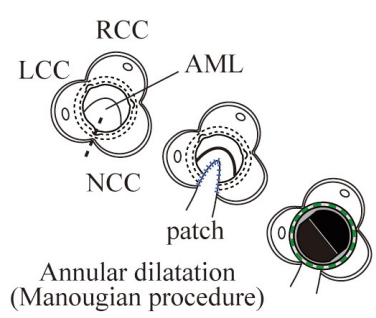

B

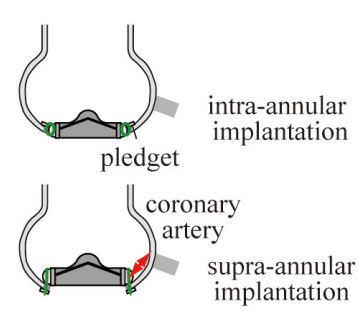

C
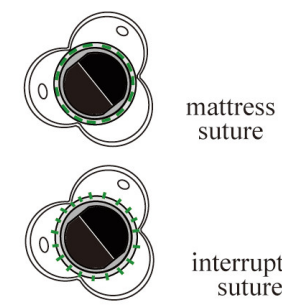

suture

interrupted suture

Figure 4. Solutions for small aortic annulus. A: Annular dilation by Manougian procedure. The commissure between LCC and NCC is incised and a patch is placed. B: Supra-annular implant. Care should be taken to avoid an obstruction of coronary artery. C: Interrupted suture. Cutting of annular tissue while tying is a pitfall. 


\subsubsection{Supra-annular implantation}

This allows implantation of a prosthetic valve that is one size larger and is commonly used in many institutes (Fig. 4B). A non-everting suture is placed along the annulus. However, this generates a risk of occlusion of the coronary ostium when it is located at an unusually low level. It can be evaluated by CT, but may be missed by coronary angiography. Intraoperative TEE is helpful for making a decision (Fig. 2A).

\subsubsection{Interrupted suture}

Although mattress sutures slightly reduce the circumference of the aortic annulus, interrupted sutures allow insertion of a prosthetic valve with the same size as the annulus (Fig. 4C). This slightly increases the number of sutures required. Since appropriate seating of the sewing ring on the annulus cannot be confirmed by the pledget adjacent to the sewing cuff, it needs to be inspected through the valve by manually opening the leaflet. When the annulus is severely calcified, it is important to prevent a gap between the annulus and sewing ring. Furthermore, cutting of annular tissue should be avoided while the suture is tied. Two other solutions are stentless aortic valve implant $[12,13]$ and apicoaortic conduit [14-16]. The reader should consult the literature for further details on these two methods.

\section{Calcified aorta}

Calcification in the aorta occasionally necessitates modification of the surgical strategy because of the safety of arterial cannulation, aortic cross-clamping, insertion of a root cannula, aortotomy and suture closure of the aorta. Calcification can be precisely assessed by preoperative CT. The principal strategy can be selected based on the CT findings. Intraoperative TEE and/or epiaortic echo may be used to identify the precise location and extent of calcification and atheromatous plaque in the surgical field. Echo-guided marking of calcification on the aorta can be helpful for subsequent surgical procedures. Three-dimensional (3D) TEE is capable of visualizing the entire ascending aorta (Fig. 5). The xPlane mode allows serial sections of the aorta to be scanned from the aortic valve to the aortic arch. The diseased portion of the aorta can be located by digital compression of the aorta during visualization by TEE.

Fig. 6 demonstrates several TEE images of calcified aortas. Fig. 6A shows a calcified aortic wall just distal to the sinotubular junction, accompanied by acoustic shadow. The aortotomy line needs to be shifted distally. Calcification can take place at the level of the aortic cross-clamp, where the right pulmonary artery crosses behind the ascending aorta (Fig. 6B). Fig. 6C shows calcification at the level of arterial cannulation. The use of epiaortic echo is desirable to accurately locate the site of calcification. Fig. 6D is a TEE image of rather mild calcification. In this case, the aorta could be clamped without cerebral complications. Fig. 6E shows atheromatous plaque on the anterior wall of the ascending aorta at the level of cross-clamping and insertion of the root cannula. Since enhanced CT is not often employed for preoperative assessment before aortic valve replacement, care should be taken to avoid missing such 
findings. Palpation of the aorta cannot detect the presence of atheroma. TEE or epiaortic echo is needed to avoid an embolic event.

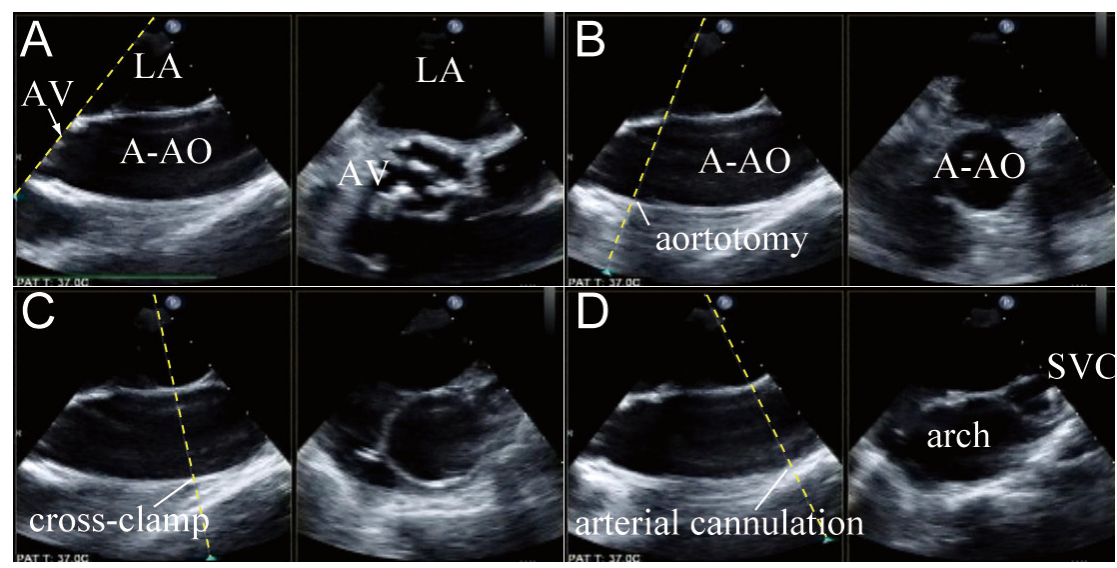

Figure 5. Scanning the entire A-AO using xPlane mode. In the midesophageal A-AO long-axis view, the scanning plane orthogonal to the reference plane is tilted from the AV up to the arch level. Short-axis view of A-AO can be visualized.

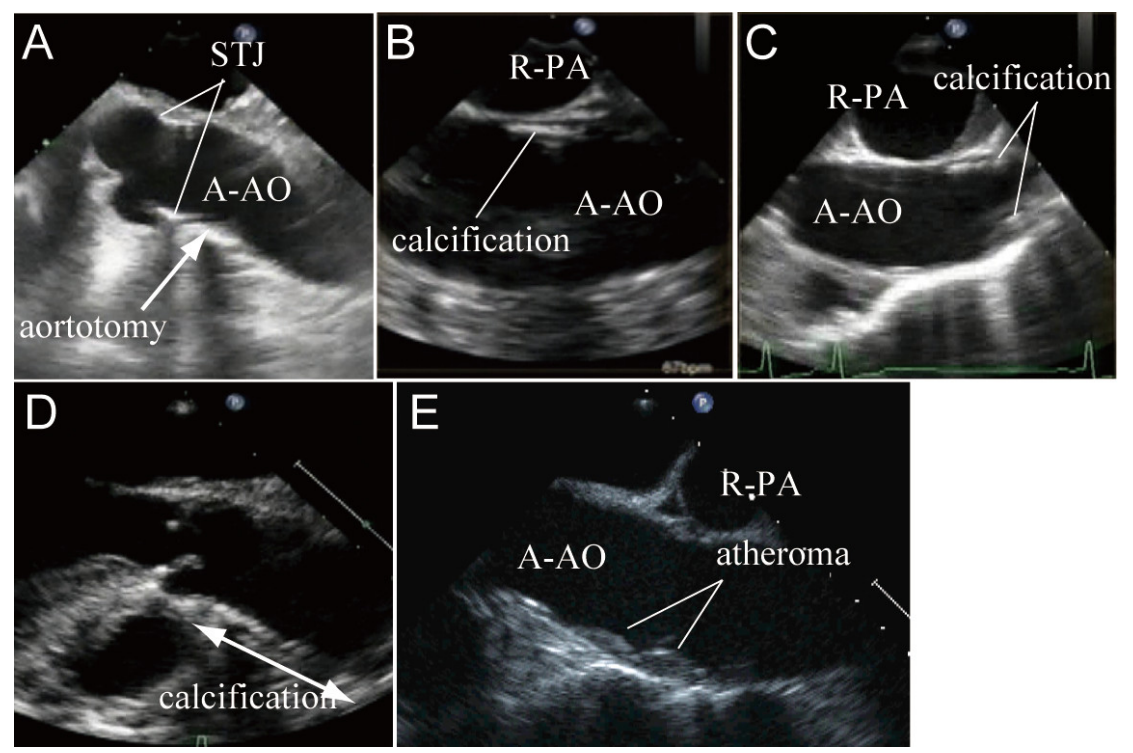

Figure 6. Examples of TEE images of calcified A-AO. A: Calcification of anterior wall just distal to STJ. B: Calcification at the level of cross-clamping (R-PA level). C: Calcification at the arterial cannulation level. D: Mild calcification of A-AO. E: Atheromatous plaque in the A-AO. 


\subsection{Arterial cannulation and insertion of a root cannula}

Calcification often involves the distal portion of the ascending aorta and makes arterial cannulation difficult (Fig. 6C). Since an adequate space for aortotomy needs to be spared for procedures on the aortic valve, the cannulation site should not be too proximal. Arterial cannulation of a calcified aorta might cause aortic dissection at the time of cannulation and/or difficult hemostasis following decannulation. When aortic cannulation is not desirable, the axillary or femoral artery can be used as an alternative perfusion route. However, in patients with a calcified aorta, there can also be stenosis or atheromatous lesions in the innominate or iliac artery; thus, it is mandatory to check for these lesions before surgery. The insertion site of the root cannula should be carefully determined. Epiaortic echo is helpful for examining the presence and location of calcification and atheroma. A safe placement of the root cannula requires a certain length of nearly normal wall, which allows secure closure of the puncture site. When an adequate area is not available, an elastic needle may be used instead.

\subsection{Aortic cross-clamp}

In cases with severe and diffuse calcification of the aorta (porcelain aorta), concomitant replacement of the ascending aorta should be considered [17-19]. However, in the majority of calcified aortic valve cases, calcification is present but not severe; it is often scattered without apparent atheromatous plaque on the intima. It is important to determine the safety of aorta cross-clamp in all cases, since cross-clamp of a calcified aorta potentially causes fracture of the calcified layer that can lead to aortic dissection or embolism of fragmented calcium.
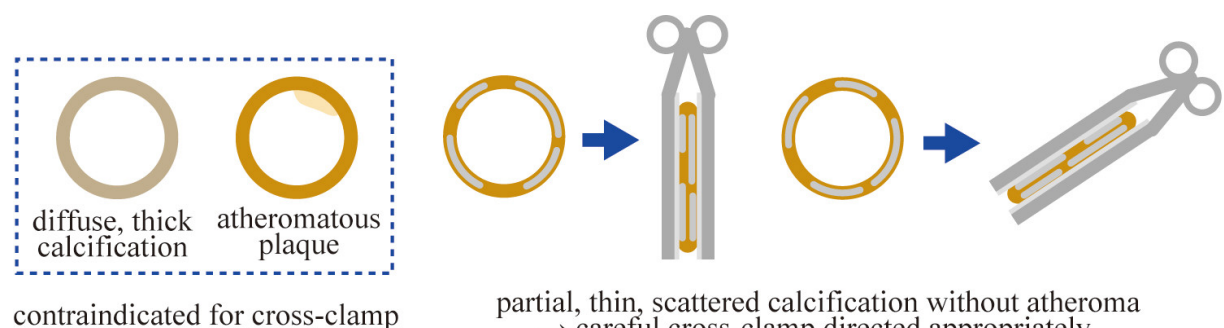

partial, thin, scattered calcification without atheroma $\rightarrow$ careful cross-clamp directed appropriately

Figure 7. Strategies for clamping in cases of calcified aorta. When calcification is diffuse and thick or atheromathous plaque is present, cross-clamp is avoided. If calcification is partial and thin without atheroma, the aorta can be clamped according to the distribution of calcified portion.

Fig. 7 shows the strategy of the author. When calcification is diffuse and thick or atheromatous plaque is present, aortic cross-clamp is avoided. If the calcified layer is partial and not thick, the aorta may be clamped with some fracture of the calcified portion, but without incompetent clamp or the development of dissection. The clamp may be applied as parallel to the calcified wall as possible to minimize fracture of the calcified section and the shear force on the intima. Initially, the ratchet is locked slowly and less deeply. The blood flow in the proximal aorta is checked with TEE before antegrade cardioplegia is given. When there is some leak through 
the clamp site, the ratchet is advanced. If full clamp is not effective, another straight clamp is added adjacent to the first clamp or conversion to aortic repair is considered, depending on the risk of aortic repair in each individual case. It should be kept in mind that a clamp on the calcified aorta flattens the proximal aorta and narrows the surgical field following aortotomy. In the case shown in Fig. 6D, the aorta was carefully clamped and valve replacement was performed without neurologic sequelae. For porcelain aorta, endovascular clamping may be another option [20].

\subsection{Aortotomy}

An incision line is determined based on the distribution of calcification. It may be modified from the standard J-shaped incision line to a rather transectional or more oblique and distal incision. In the case shown in Fig. 6A, aortotomy at a more distal level or meticulous removal of calcification is needed. If there is an insufficient margin for suture closure of the aortotomy (at least $1 \mathrm{~cm}$ in width), the surgeon should be prepared for difficult suturing. When the calcified portion of the aorta is to be incised and sutured, calcium needs to be carefully removed following aortotomy, but before implanting a prosthetic valve. The use of CUSA is helpful for reducing the amount of calcium, enabling an adequate attachment of the aortic walls during suture closure. A new device for this purpose may be helpful [21].

\section{Coronary arteries}

Myocardial protection is one of the most important issues in aortic valve replacement. Left ventricular hypertrophy and/or coronary artery disease is often associated with aortic stenosis. In addition, surgical procedures may potentially cause new myocardial ischemia. Therefore, careful observation of coronary perfusion is essential. Fig. 8 shows visualization of the coronary arteries with TEE. The coronary arteries can be visualized bilaterally in the majority of cases and coronary artery assessment is done routinely in our institute. The ostia of the right and left coronary arteries are depicted in the short-axis view at the level of sinus of Valsalva (Fig. 8 A,B). The right coronary artery travels in the groove between the right atrium and right ventricle, whereas the left coronary artery crosses behind the pulmonary artery. The left main truncus divides into the left anterior descending and left circumflex arteries. The right coronary ostium can also be depicted in the midesophageal aortic valve long-axis view, giving an idea of its distance from the annulus (Fig. 8C).

To further visualize the distal portion of the left coronary artery, the scanning plane is rotated counterclockwise from the midesophageal aortic valve long-axis view (Fig. 8D). The left coronary sinus takes the place of the noncoronary sinus, and the short-axis view of left main truncus appears. The left anterior descending artery courses toward the 6 to 7 o'clock direction. The distal portion of the left circumflex artery is visualized in the atrioventricular groove. With the 3D en face view, the height of coronary take-off can be observed (Fig. 8E). The author meticulously uses TEE for intraoperative assessment of coronary perfusion, because it is the 
most important factor that affects the outcomes of aortic valve replacement, especially for calcified aortic valves. Several pitfalls related to myocardial ischemia are shown in Fig. 9.

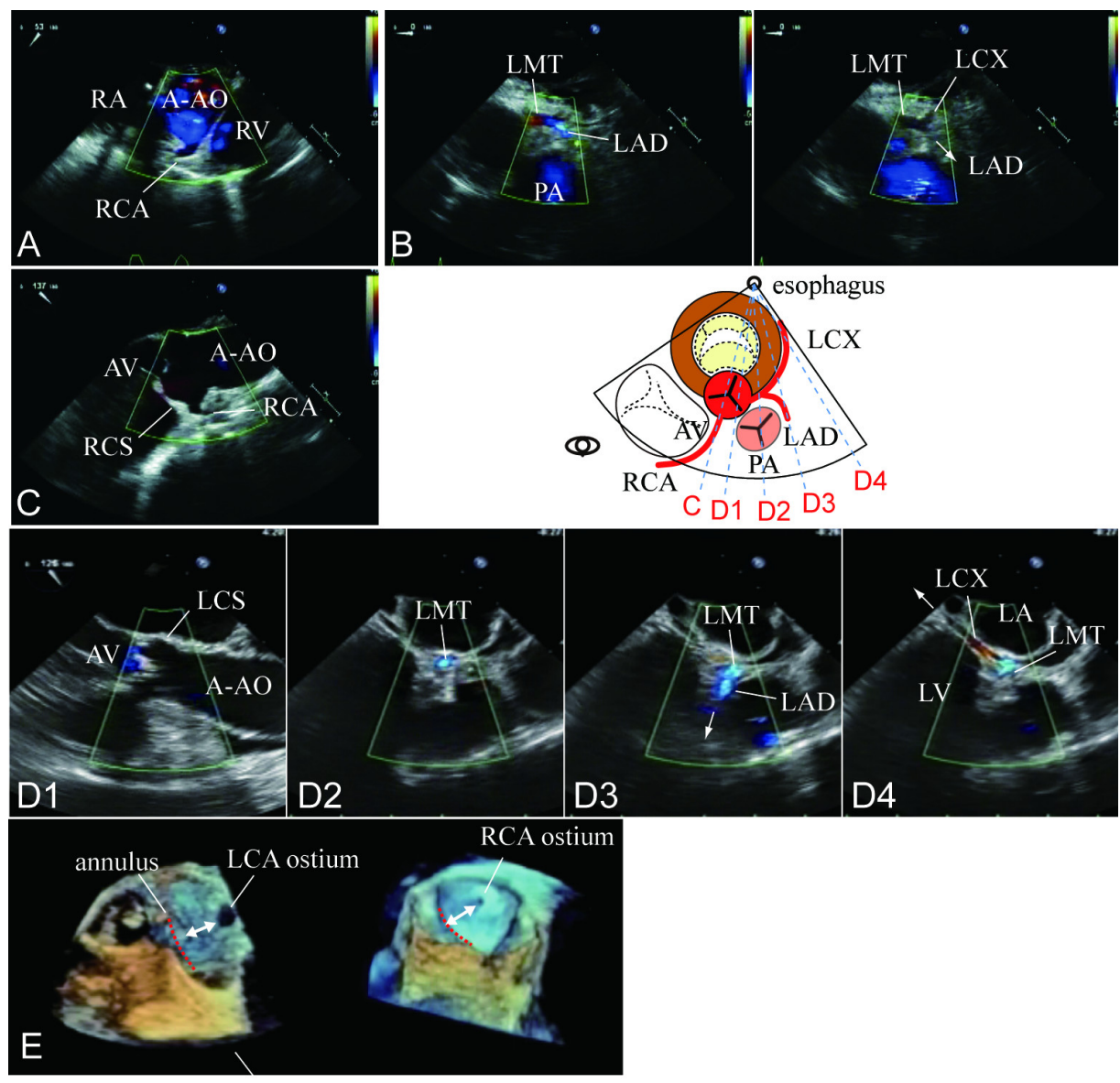

Figure 8. Visualization of coronary arteries with TEE. A: In short-axis view at the level of sinus of Valsalva, RCA arises from RCS at 6 o'clock position directed to the groove between RA and RV. B: The LCA takes off from LCS at 3 o'clock position. LMT courses behind PA and divides to LAD and LCX. C: In midesophageal AV long-axis view, RCA arises from RCS (C). D: As the probe is rotated counterclockwise, LCA arises from LCS and LMT divides to LAD and LCX. LAD is directed toward the LV apex and LCX courses posteriorly along the atrioventicular groove between LA and LV. E: In 3D en-face view, distance from AV annulus to the ostia is recognized. 

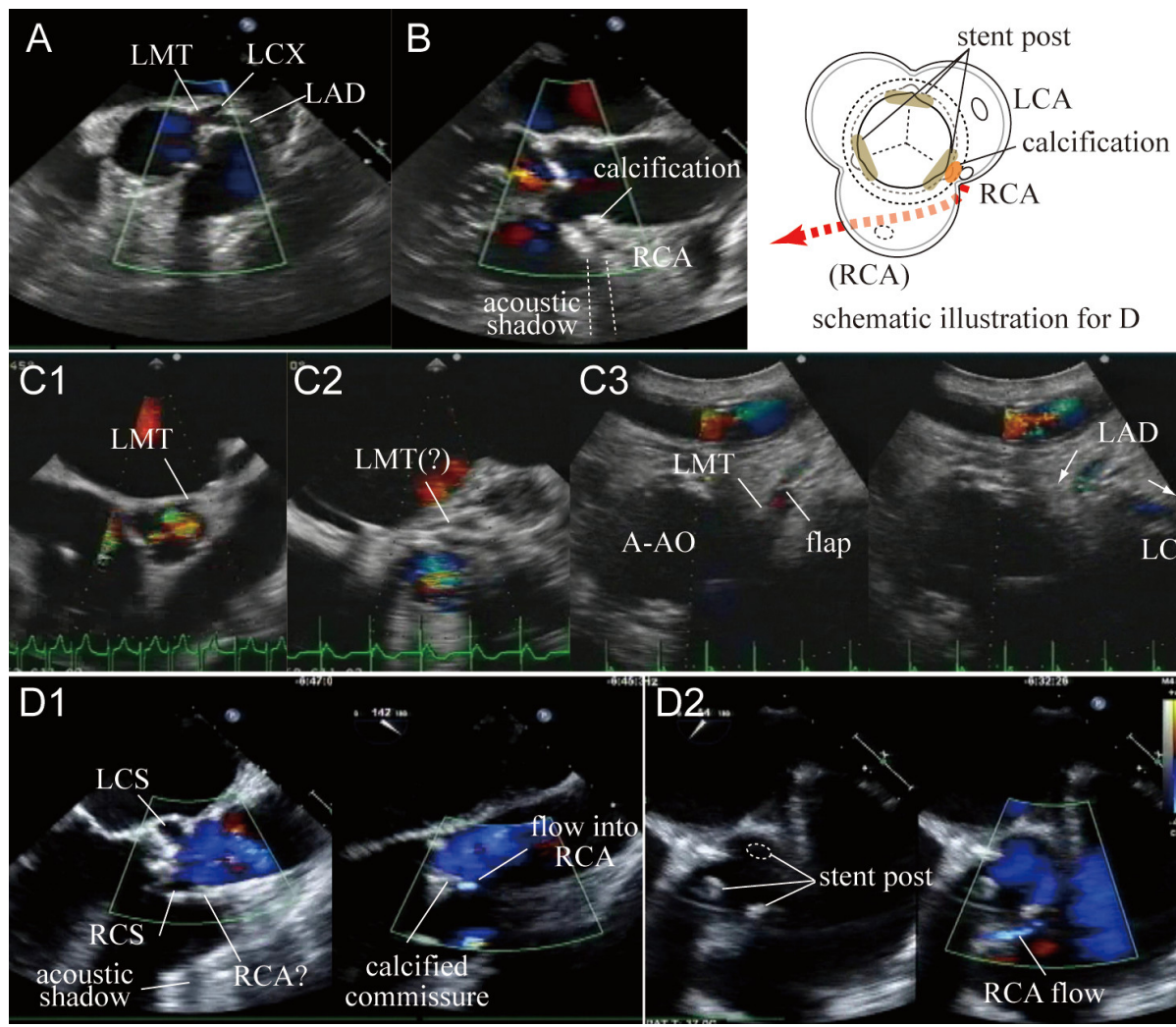

schematic illustration for D
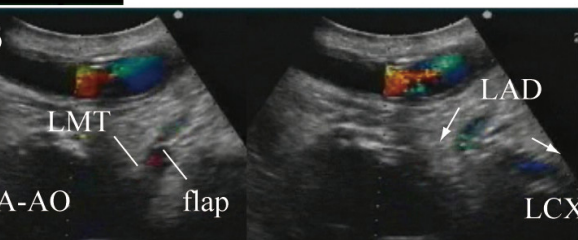

Figure 9. Pitfalls related to coronary artery in aortic valve replacement. A: Short LMT divides to LAD and LCX near the ostium. B: Calcification adjacent to RCA ostium. C: New dissection in the LMT. LMT was intact before bypass (C1), but no flow was detectable during wearing from bypass (C2) associated with akinetic LV. Epicardial echo following coronary bypass to the LAD shows a flap obstructing the LMT and blood flow from LAD toward LCX (C3). D: Anomalous origin of RCA. RCA was not found in the RCS, but at the commissure between RCC and LCC (D1). Following valve replacement, RCA was seen originating from the commissure in front of the stent post (D2). Schematic illustration of $D$ is shown.

\subsection{Short left main truncus}

The left main truncus is occasionally found to be short (Fig. 9A). As the cannula is forcefully thrust into the ostium, the cannula tip may enter either the left anterior descending or left circumflex artery. Any unperfused region of the left ventricle may be poorly protected during the procedure.

\subsection{Calcified ostium}

The coronary ostium as well as the surrounding wall of the coronary sinus of Valsalva may be rigid or irregular, occasionally making it difficult to selectively infuse cardioplegic solution 
into the coronary artery, because the cannula tip does not fit properly and there is significant leakage. When calcification accompanied by acoustic shadow is present adjacent to the coronary ostium (Fig. 9B), this pitfall may occur. A highly echogenic projection accompanied by acoustic shadow, usually on the side of the sinotubular junction, is a typical TEE finding. Calcification in the aortic root also makes it difficult to locate the right coronary ostium, especially when the sinus wall of Valsalva is rigid and cannot be folded back. Unsuccessful infusion of cardioplegic solution potentially leads to right heart failure, since the right coronary region cannot be adequately perfused via the retrograde approach. Although a large-bore cannula tip does not adequately fit the calcified ostium, a small-caliper cannula causes little leakage but the velocity of jet stream ejected out of the cannula can exceed $2 \mathrm{~m} / \mathrm{sec}$, potentially causing intimal damage or dissection. Fig. 9C shows the TEE findings of dissection that occurred in the left coronary artery following cardiopulmonary bypass in a case of difficult coronary perfusion. Although the left coronary artery was intact before bypass, no blood flow was detected during weaning from bypass in the area that was thought to contain the left main truncus. Since the anterior and posterior walls of the left ventricle were akinetic, immediate coronary bypass grafting to the left anterior descending artery was performed. Subsequent epicardial echo revealed a flap in the left main truncus and retrograde blood flow in the left anterior descending artery from the coronary bypass graft that perfused the left circumflex artery.

\subsection{Anomalous origin of the right coronary artery}

Anomalous origin of the right coronary artery is rather rare (Fig. 9D), but is difficult to diagnose based on preoperative coronary angiography. In a case of aortic stenosis with an intact right coronary artery in preoperative coronary angiography, the right coronary ostium could not be found in the right coronary sinus of Valsalva. Selective perfusion of the left coronary artery caused backflow from the right coronary ostium, which was identified at the commissure between the right and left coronary cusps. Calcified protrusion was continuous from the commissure to the ostium. The right coronary artery was successfully perfused, and the calcium at the orifice was left unresected. However, when calcium is exposed without being covered by the sewing ring, there is the potential for thrombus formation adjacent to the right coronary ostium. Following aortic valve replacement, TEE demonstrated that the stent post was located in front of the right coronary ostium, because the stent posts were oriented so that the left coronary ostium was not covered by the stent post. The right coronary artery coursed along the right coronary sinus and could have been inadvertently injured by the surgical procedures.

\subsection{Myocardial perfusion during cardioplegia}

Retrograde cardioplegia is a solution for difficult antegrade coronary perfusion. However, the region perfused by the right coronary artery is poorly protected because the cardioplegic solution predominantly enters the coronary veins along the left coronary artery (great cardiac vein) but not the middle or small cardiac veins. Although the latter may be perfused via collaterals between the veins, this is not certain. Also, in cases where it is difficult to fit the 
coronary perfusion cannula and there is considerable leakage, perfusion of the myocardium is a concern. A myocardial thermometer was conventionally used, but may not be useful in warm heart surgery. Real-time assessment of myocardial perfusion is desired, especially in cases with deteriorated cardiac function. Here, a novel method for noninvasively assessing myocardial perfusion is demonstrated (Fig. 10). The transgastric basal or midventricular shortaxis view is visualized. In pulsed-wave Doppler mode, the sample volume is placed on the myocardium. When this portion of myocardium is perfused, a flow signal is detected. Under selective perfusion of the left coronary artery, blood flow is detected in the anterior wall but not in the inferior wall. As coronary perfusion is discontinued, the flow signal instantaneously disappears. The perfused region is identified by mapping the myocardium. This can be helpful for examining the extent of retrograde delivery of cardioplegic solution.

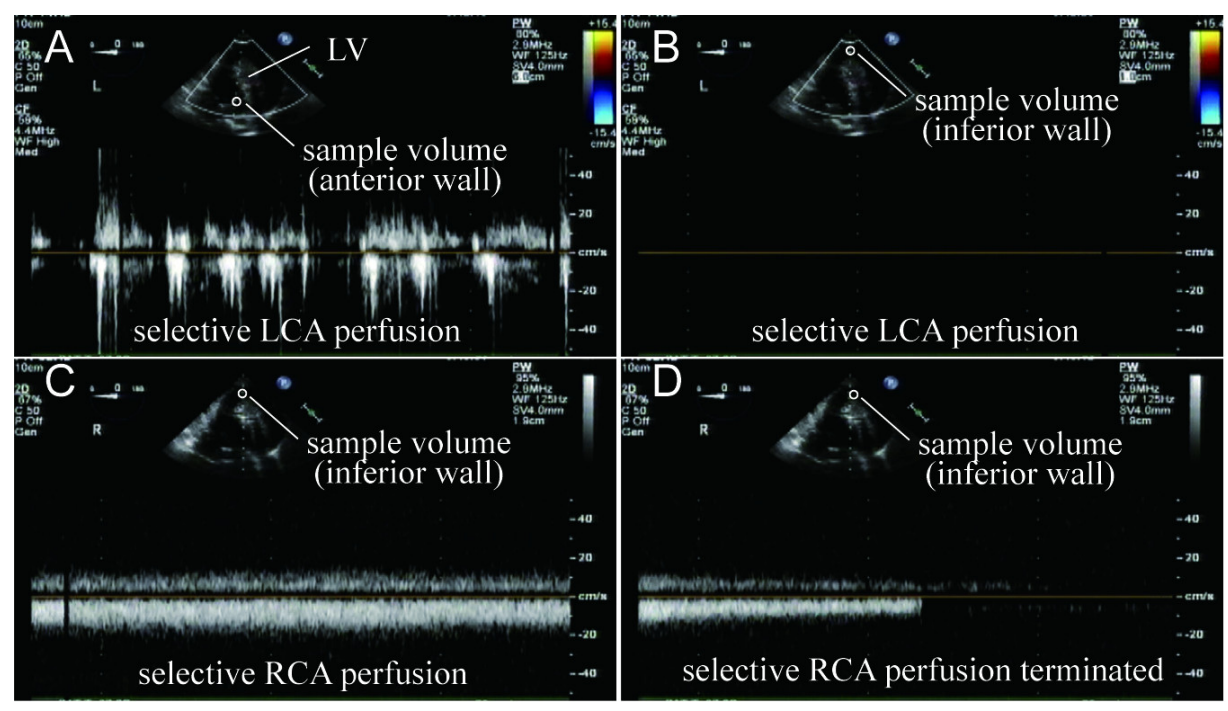

Figure 10. TEE assessment of myocardial blood flow during cardioplegia. Under selective perfusion to LCA, blood flow is detectable in the anterior wall (A) but not in the inferior wall (B). As selective RCA perfusion is terminated, blood flow detected in the inferior wall (C) instantaneously disappears (D)

\section{Assessment following implantation}

After the aortotomy is closed, several pitfalls need to be checked (Fig. 11,12). If an unexpected event is detected, a prompt decision for additional intervention should be made.

\subsection{Perivalvular or transvalvular leakage}

During weaning from bypass, the surgeon should check for perivalvular leakage. A calcified aortic valve can result in inadequate contact between the annulus and the suture ring of the 
prosthetic valve. It is readily examined by TEE in the midesophageal long-axis view (Fig. 11A). A minor leak that originates inside of the ring and deviates inwards is transvalvular leak and is not significant (Fig. 11B). When significant leakage is detected in the left ventricular outflow tract that originates outside of the suture ring, perivalvular leakage is probable (Fig. 11C). The assessment of leakage is difficult in cases with concomitant mitral valve replacement. The ring of the prosthetic valve implanted in the mitral position casts an acoustic shadow on the left ventricular outflow tract (Fig. 11D). In such an instance, leakage is assessed in the deep transgastric long-axis view via the left ventricular apex (Fig. 11E).
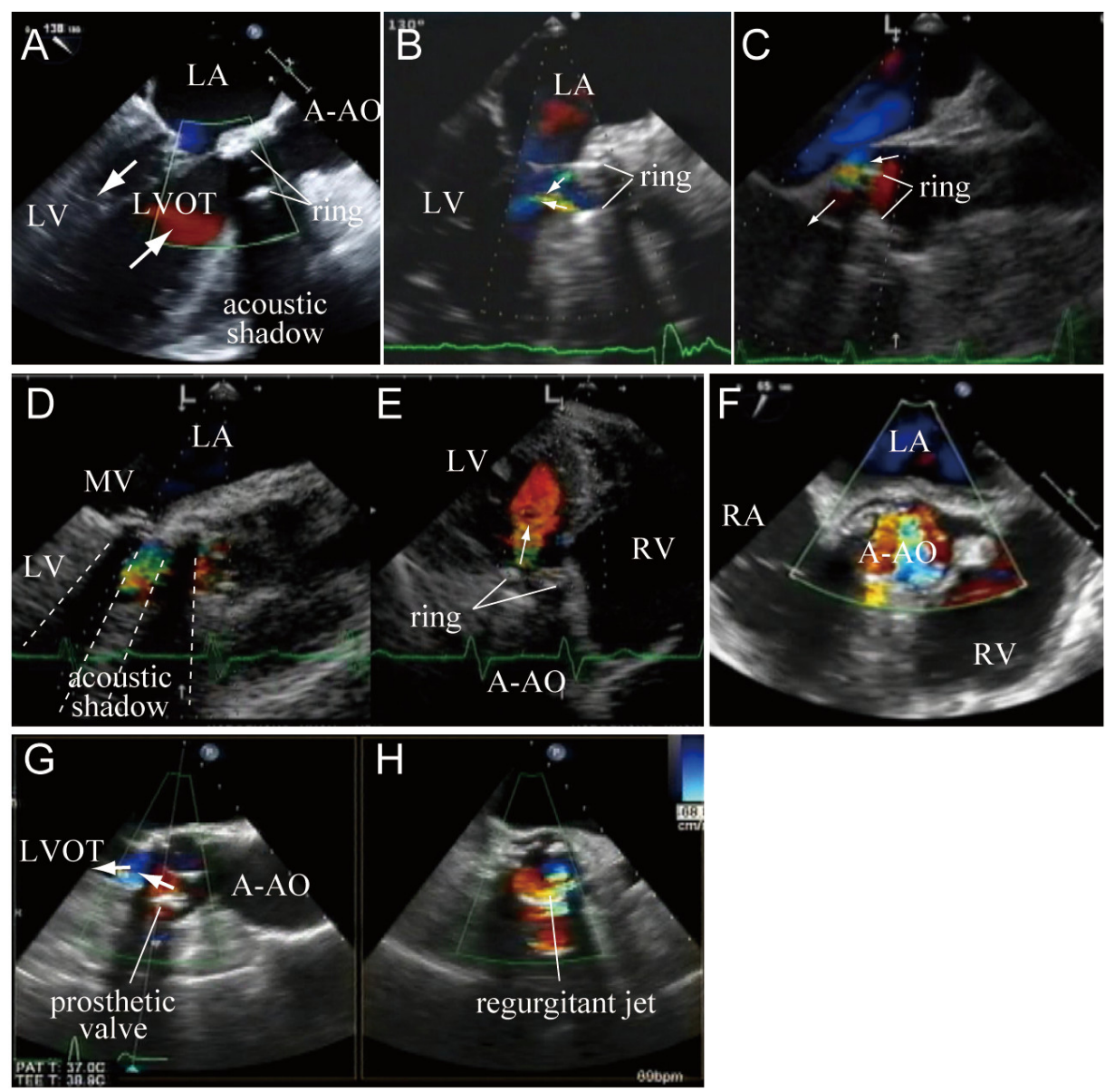

Figure 11. TEE assessment of prostethic valve following aortic valve replacement. A: Midesophageal long-axis view without abnormal leak from the prosthetic valve. B: Minor transvalvular leak. C: Significant perivalvular leak around the valve. D: Leakage is suspected but visualization is disturbed by the valve in mitral position. E: Deep transgastric view shows mild transvalvular leak F: Blood flow in the entire area of ascending aorta just distal to the prosthetic valve indicates good opening of discs. G,H: Massive transvalvular leak from the bioprosthesis due to everted leaflet. 
Valve dysfunction should be checked during weaning from bypass. Dysfunction of a mechanical valve includes an immobilized disc at the open or closed positions. Since the disc itself is hardly depicted by TEE, dysfunction is examined in the color Doppler mode. In the short-axis view of the ascending aorta at the level just above the valve, opening of both discs can be confirmed by the flow signal, which fills the entire area of the aorta (Fig. 11F). Incompetence of the valve is caused by an immobilized disc of a mechanical valve at the open position probably due to debris or calcium between the disc and ring or by jammed leaflets of a bioprosthetic valve. It can be recognized by massive aortic regurgitation, demonstrated by a regurgitant jet in the left ventricular outflow tract originating from inside of the ring in midesophageal or deep transgastric view (Fig. 11G,H). In this case, exploration revealed that there was unintended eversion of a leaflet on the noncoronary side without jamming or leaflet damage. The leaflet was returned to the normal position and the aortotomy was closed [22].

\subsection{Coronary ostium and ventricular contraction}

Obstruction of coronary ostium is checked during weaning from bypass to minimize myocardial damage under warm ischemia. This event is important especially for the valve implanted in the supra-annular position. In the early timing following aortic declamping, patency and blood flow in both coronary arteries is checked. Both ostia can be visualized in the short-axis view just above the valve (Fig. 12A). In the midesophageal aortic valve long-axis view, the right coronary ostium is seen above the sewing ring (Fig. 12B). The presence of flow and distance from the ring of the prosthetic valve is checked.

Blood flow in the left coronary artery is examined proximal to its bifurcation into the LAD and LCX as shown in Fig. 8. Fig. 12D shows TEE views in a case of a low origin of the LMT. Before cardiopulmonary bypass, the midesophageal long-axis view showed a short distance between the aortic annulus and left main truncus. Following valve replacement at the intra-annular position, knots were found to be in front of the left coronary ostium and accelerated flow was seen. In the midesophageal long-axis view, the left coronary ostium was just above the ring. Surprisingly, the left main truncus was just behind the annulus. A deep suture could have injured the left main truncus. Fig. 12E and F demonstrate a case with a normal origin of the main left coronary artery. Before cardiopulmonary bypass, the coronary orifice was approximately $1 \mathrm{~cm}$ above the aortic annulus (Fig. 12E1,2). The 3D en face view clearly demonstrated an adequate distance (Fig. 12 E3). After replacement, TEE showed that there was no obstacle in front of the left main truncus in the short-axis view (Fig. 12F1). In the long-axis view, there was adequate distance between the ring and left main truncus (Fig. 12F2). 3D TEE also clearly showed safe implant of the prosthetic valve (Fig. 12F3). As ventricular contraction recovers, regional wall motion abnormalities can be assessed in the midesophageal, 2-chamber and 4chamber views, or the transgastric basal or mid-short-axis views.

If coronary obstruction is suspected as in Fig. 9, early coronary revascularization is to be considered for minimizing myocardial damage. Ischemia can develop not only by obstruction of coronary ostium but by poor myocardial protection or embolism of dislodged calcium or even intracardiac air. Sustained circulatory support may be advantageous or retrograde cardioplegia may potentially help to displace any calcium or air embolus toward the coronary ostium. 

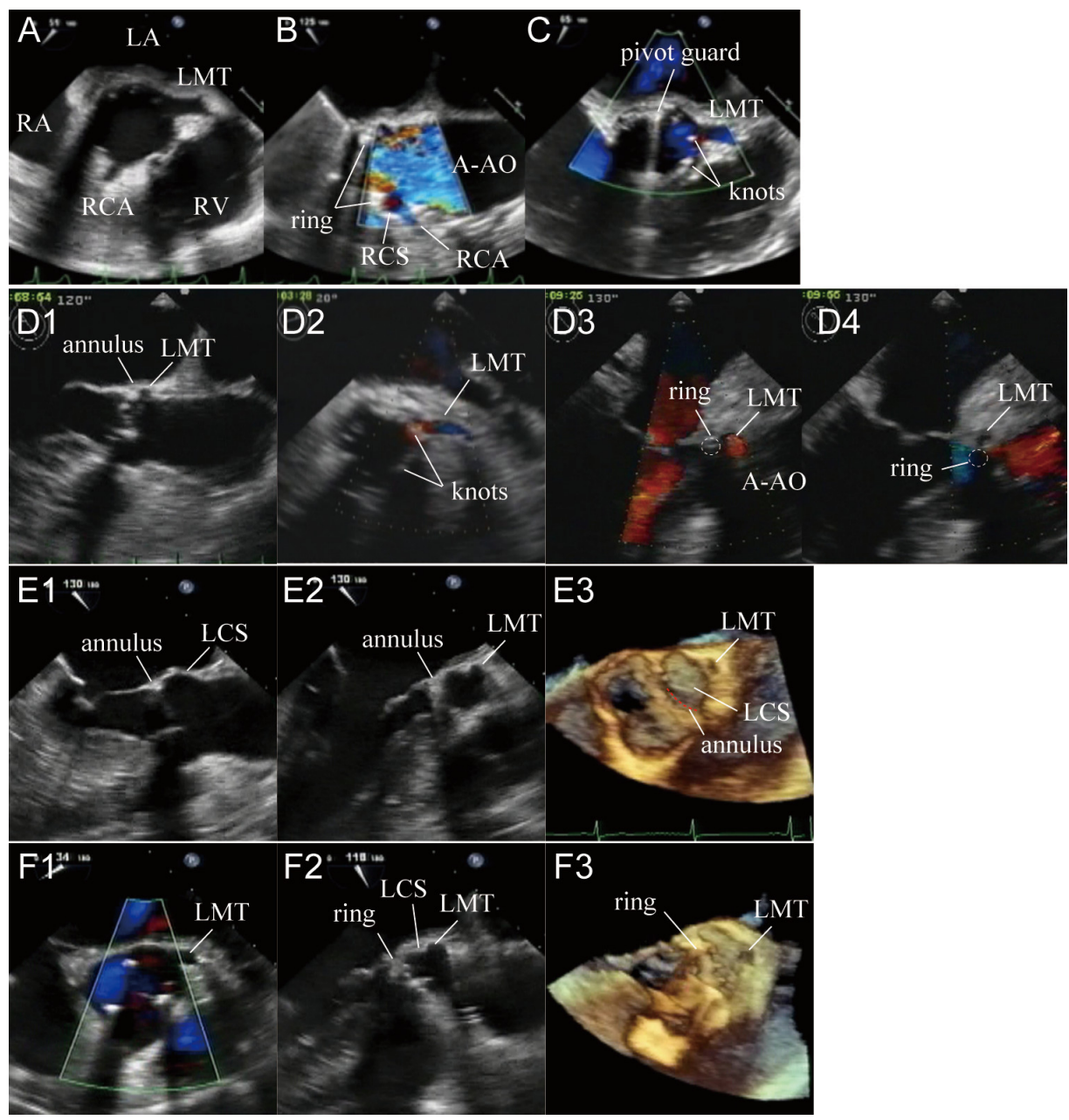

Figure 12. TEE assessment of coronary arteries in aortic valve replacement. A; Short-axis view at the level of the sinus of Valsalva that shows patent ostia of the RCA and LMT. B: RCA flow in midesophageal AV long-axis view. Distance from the ring to RCA ostium is checked. C: LMT flow in short-axis view of sinus of Valsalva. Knots are seen without obstruction. D: Low take-off of LMT (D1). Following valve replacement, the knots are in front of LMT ostium. A mild acceleration of blood flow is seen (D2). In midesophageal long-axis view, the LMT orifice is just above the ring (D3). LMT was found to course very close to the suture (D4). E: Normal take-off of LMT. LMT originates from the LCS at approximately $1 \mathrm{~cm}$ above the aortic annulus (E1 to E2). 3D en face view clearly demonstrates the distance of LMT from the annulus (E3). F: TEE views following valve replacement. There is no obstruction in front of the LMT (F1). There is adequate distance from the ring to the LMT orifice, depicted in midesophageal long-axis view (F2) and 3D en face view (F3)

\subsection{New mitral regurgitation}

Mitral regurgitation may appear following aortic valve replacement. One reason is perforation of the mitral annulus caused by excessive decalcification of the aortic annulus. Another 
mechanism is systolic anterior motion of the mitral leaflet, which is caused by reduced left ventricular dimensions and/or a sigmoid septum due to left ventricular hypertrophy. When several measures are not effective, emergent mitral valve replacement should be considered.

\subsection{Aortic dissection}

Following aortic declamping, the aorta distal to the clamp site should be checked for new dissection. It may potentially develop due to detachment of the calcified aortic wall, and inner or outer layer. Dissection can also originate from the arterial cannulation site, root cannula site or aortotomy site.

In conclusion, it is essential to watch for pitfalls in aortic valve replacement for calcified aortic valve, because it is commonly associated with various pathologies that can affect the surgical outcomes. Meticulous monitoring and intraoperative diagnostic imaging are helpful for achieving the best possible results in cases with increased risk factors.

\section{Abbreviations for figures}

A-AO ascending aorta

AML anterior mitral leaflet

AV aortic valve

LA left atrium

LAD left anterior descending artery

LCA left coronary artery

LCC left coronary cusp

LCS left coronary sinus

LMT left main truncus

LVOT left ventricular outflow tract

MV mitral valve

NCC noncoronary cusp

PA pulmonary artery

RA right atrium

RCA right coronary artery

RCC right coronary cusp

RCS right coronary sinus 
R-PA right pulmonary artery

RV right ventricle

STJ sinotubular junction

SVC superior vena cava

\section{Author details}

Kazumasa Orihashi

Department of Cardiovascular Surgery, Kochi Medical School, Kochi, Japan

\section{References}

[1] Tanaka, K, Tajima, K, Takami, Y, et al. Early and late outcomes of aortic valve replacement in dialysis patients. Ann Thorac Surg (2010). , 89, 65-70.

[2] Kaden, J. J, Eckert, J. P, Poerner, T, et al. Prevalence of atherosclerosis of the coronary and extracranial cerebral arteries in patients undergoing aortic valve replacement for calcified stenosis. J Heart Valve Dis (2006). , 15, 165-8.

[3] Staico, R, Armaganijan, L, \& Lopes, R. D. Coronary embolism and calcified aortic valve: is there a correlation? J Thromb Thrombolysis (2012). , 34, 425-7.

[4] Mannino, G, Romano, M, Calanchini, M, Mannino, C, \& Cascone, N. C. Branch retinal artery embolization due to calcific aortic valve stenosis. Eur J Ophthalmol (2010). , 20, 625-8.

[5] Mahajan, N, Khetarpal, V, \& Afonso, L. Stroke secondary to calcific bicuspid aortic valve: case report and literature review. J Cardiol (2009). , 54, 158-61.

[6] Rivard, A. L, Bartel, T, Bianco, R. W, et al. Evaluation of aortic root and valve calcifications by multi-detector computed tomography. J Heart Valve Dis (2009). , 18, 662-70.

[7] Aviram, G, Sharony, R, Kramer, A, et al. Modification of surgical planning based on cardiac multidetector computed tomography in reoperative heart surgery. Ann Thorac Surg (2005). , 79, 589-95.

[8] Islamoglu, F, Apaydin, A. Z, Degirmenciler, K, et al. Detachment of the mitral valve anterior leaflet as a complication of aortic valve replacement. Tex Heart Inst J (2006). , 33, 54-6. 
[9] Orihashi, K. Intraoperative imaging in aortic valve surgery as a safety net (Chapter 1). IN: Motomura N, ed. Aortic Valve Surgery. InTech Co., Croatia, (2011). , 3-18.

[10] Taniguchi, S, Noguchi, M, Onohara, D, et al. Aortic valve replacement with 17-mm St. Jude Medical Regent prosthetic valves for a small calcified aortic annulus in elderly patients. Gen Thorac Cardiovasc Surg (2010). , 58, 506-10.

[11] Coutinho, G. F, Correia, P. M, Paupério, G, et al. Aortic root enlargement does not increase the surgical risk and short-term patient outcome? Eur J Cardiothorac Surg (2011). , 40, 441-7.

[12] Karimov, J. H, Cerillo, A. G, Solinas, M, et al. Stentless aortic valve implantation in heavily calcified aorta. J Cardiovasc Med (Hagerstown) (2009). , 10, 813-4.

[13] Di Matteo G Masala N, Swanevelder J, et al. Clinical outcome of a simplified technique for aortic valve replacement with stentless bioprostheses. J Heart Valve Dis (2009). , 18, 111-8.

[14] Shin, H, Mori, M, Suzuki, R, et al. Apicoaortic valved conduit with an apical connector for aortic stenosis with a calcified aorta. Gen Thorac Cardiovasc Surg (2009). , 57, 467-70.

[15] Chahine, J. H, Rassi, I, \& Jebara, V. Apico-aortic valved conduit as an alternative for aortic valve re-replacement in severe prosthesis-patient mismatch. Interact Cardiovasc Thorac Surg (2009). , 9, 680-2.

[16] Crestanello, J. A, Zehr, K. J, Daly, R. C, et al. Is there a role for the left ventricle apical-aortic conduit for acquired aortic stenosis? J Heart Valve Dis (2004). , 13, 57-62.

[17] Chung, S, Park, P. W, Choi, M. S, et al. Surgical experience of ascending aorta and aortic valve replacement in patient with calcified aorta. Korean J Thorac Cardiovasc Surg (2012). , 45, 24-9.

[18] Iliopoulos, D. C, Deveja, A. R, Satratzemis, V, et al. Deep hypothermic arrest for aortic valve replacement in case of porcelain aorta. Asian Cardiovasc Thorac Ann (2009). , 17, 415-6.

[19] Okamoto, H, Fujimoto, K, Tamenishi, A, et al. Aortic valve replacement in a heavily calcified "porcelain" aorta. Jpn J Thorac Cardiovasc Surg (2001). , 49, 453-6.

[20] Ooi, A, Iyenger, S, Langley, S. M, et al. Endovascular clamping of porcelain aorta in aortic valve surgery using Foley Catheter. Heart Lung Circ (2006). , 15, 194-6.

[21] Kudo, M, Misumi, T, \& Koizumi, K. Aortotomy and endarterectomy of the ascending aorta for aortic valve replacement in a patient with porcelain aorta. Surg Today (2005). , 35, 1000-3.

[22] Orihashi, K, Kurosaki, T, \& Sueda, T. Everted leaflet of a bovine pericardial aortic valve. Interact Cardiovasc Thorac Surg (2010). , 10, 1059-60. 